Nouvelles perspectives en sciences sociales

Revue internationale de systémique complexe et d'études relationnelles

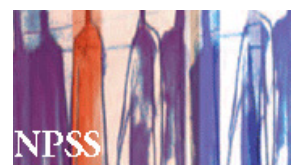

\title{
Échanges, contrats et diversité des cultures
}

\section{Philippe d'Iribarne}

Volume 9, numéro 2, mai 2014

Sur le thème de l'échange

URI : https://id.erudit.org/iderudit/1025975ar

DOI : https://doi.org/10.7202/1025975ar

Aller au sommaire du numéro

\section{Éditeur(s)}

Prise de parole

ISSN

1712-8307 (imprimé)

1918-7475 (numérique)

Découvrir la revue

Citer cet article

d'Iribarne, P. (2014). Échanges, contrats et diversité des cultures. Nouvelles perspectives en sciences sociales, 9(2), 187-203.

https://doi.org/10.7202/1025975ar

\section{Résumé de l'article}

La théorie économique des contrats suppose que leur conception et leur mise en oeuvre sont indépendantes du contexte culturel. Or, ce que les acteurs ont en tête quand ils passent des " contrats " diffère d'un pays à l'autre, en fonction de la conception d'une bonne manière de vivre et de travailler ensemble qui y prévaut. Nous en donnerons trois exemples : la variété des rapports entre une banque de développement et ses clients; la manière dont une entreprise présente ses relations avec ses clients en France et aux États-Unis; les relations entre clients et fournisseurs dans les rapports entre entreprises chinoises. Nous verrons en outre que la place qu'occupe la référence contractuelle dans le fonctionnement des entreprises et des économies est intimement liée à la vision de la société dont est porteuse la culture américaine. 


\section{Échanges, contrats et diversité des cultures}

Philippe d'IRIBARne

Gestion et Société, CNRS, Paris

$\mathrm{A}_{\text {recherche accordant une large place aux théories des incita- }}^{\mathrm{u} \text { sein de la science économique, tout un courant de }}$ tions et de l'agence a élaboré une théorie des contrats en supposant que la conception des contrats et leur mise en œuvre, comme plus largement les rapports entre les acteurs économiques, sont indépendants du contexte culturel ${ }^{1}$. En particulier, les questions que pose le caractère incomplet des contrats ainsi que ce qui touche à la construction de relations de confiance entre les contractants tend à être traité en faisant abstraction de la diversité des cultures. Or, dès que l'on rentre dans le concret de la vie des entreprises, qu'il s'agisse de relations commerciales, du montage de coopérations de longue durée dans des joint-ventures ou des rapports entre l'entreprise et son personnel, l'expérience commune montre que la manière d'établir et de gérer un contrat varie beaucoup d'un pays à l'autre. Ce n'est pas seulement que l'usage de contrats fait plus ou moins référence (il est coutumier de dire que les Américains ont la religion du contrat et que ce n'est le cas ni des Français ni des Chinois), ou encore que les cadres institutionnels (fonctionnement de la justice, rôle donné aux

$1 \quad$ Claude Ménard (dir.), Institutions, Contracts and Organizations. Perspective from New-Institutional Economics, Cheltenham (UK) et Northampton (MA), Edward Elgar, 2000. 
contrats par le droit du travail, etc.) diffèrent selon les pays; c'est que ce que les acteurs ont en tête quand ils parlent de " contrat ", négocient les termes d'un contrat et le mettent en ouvre, diffère d'un pays à l'autre.

Un programme de recherche portant sur l'encastrement culturel du fonctionnement des entreprises, qui a abordé à ce jour une cinquantaine de pays $^{2}$, a conduit, à de nombreuses reprises, à observer le fonctionnement concret de rapports contractuels et à prêter attention aux relations qui se nouent entre les acteurs à l'occasion de tels rapports. Il a amené, dans chaque cas, à mettre ce fonctionnement en rapport avec la conception d'une bonne manière de vivre et de travailler ensemble dont les acteurs étaient porteurs, conception dont il est apparu qu'elle marquait, au-delà de la vie des entreprises, l'organisation globale de la société environnante. Le présent article a pour objet, en s'appuyant sur ces recherches, d'une part de montrer à partir de quelques exemples combien la conception des rapports contractuels propre aux acteurs varie selon les cultures, et d'autre part de montrer qu'il existe un lien intime entre d'une part la place qu'occupe la référence contractuelle dans le fonctionnement des entreprises et des économies et d'autre part la vision de la société dont est porteuse la culture américaine. Compte tenu de la grande variété de conceptions de la culture que l'on trouve dans la littérature, on précisera tout d'abord quelle est celle qui est utilisée dans les recherches sur lesquelles ce texte s'appuie.

\section{Culture, univers de sens et conception du vivre ensemble}

Les conceptions les plus courantes de la culture y associent des comportements relevant de coutumes partagées au sein d'une communauté et transmises au fil du temps de génération en

$2 \quad$ Philippe d'Iribarne, L'épreuve des différences. L'expérience d'une entreprise mondiale, Paris, Seuil, coll. "La couleur des idées », 2009; Philippe d'Iribarne, La logique de l'honneur. Gestion des entreprises et traditions nationales, Paris, Seuil, 1989; Philippe d'Iribarne, Penser la diversité du monde, Paris, Seuil, 2008; Philippe d'Iribarne, Alain Henry, Jean-Pierre Segal, Sylvie Chevrier, Tatjana Globokar, Cultures et mondialisation. Gérer par-delà les frontières, Paris, Seuil, 1998. 
génération. De telles conceptions ne permettent pas de rendre compte de ce qu'on observe dans des sociétés modernes où les manières d'agir sont marquées à la fois par une grande hétérogénéité à un moment donné et par une forte évolution dans le temps. Pour rendre compte de nos observations, nous avons dû élaborer une conception de la culture $^{3}$ qui distingue deux niveaux. Un premier niveau correspond à l'existence d'images idéales de manières de vivre et travailler ensemble, associées à certaines conceptions de la liberté, de la dignité, du devoir, des processus d'arbitrage entre points de vue opposés. Ces images, et les affects qui leur sont liés, renvoient à l'existence, au sein de chaque société politique, d'un type spécifique d'inquiétudes, de craintes, associées à des situations génératrices de sentiments de mal-être, d'angoisse ou même de déréliction.

La diversité des cultures est liée au fait que ce qui est redouté et dont on cherche à s'affranchir varie sensiblement d'une société à l'autre tout en étant marqué, dans chacune d'elles, par une forte continuité dans le temps. Ainsi, dans la société américaine, la crainte fondamentale est de se trouver à la merci des actions d'autrui. Il s'agit, pour échapper à cette crainte, de rester maître de son destin. Dans la société française, ce qui est redouté pardessus tout est d'avoir à plier, par peur ou par intérêt, devant qui peut vous nuire ou vous faire bénéficier de ses faveurs. Quand on parcourt le monde, on rencontre d'autres zones de préoccupation angoissée.

Les images idéales de bonnes et de mauvaises manières de vivre ensemble qui prévalent dans une société donnée sont intimement liées au type de crainte qui la marque. Ainsi, aux États-Unis, l'idéal de relations contractuelles librement négociées entre partenaires dont les pouvoirs de négociation sont équilibrés correspond à une forme d'organisation du vivre ensemble qui permet à chacun, en lui évitant d'être engagé dans des actions auxquelles il n'a pas donné son accord, de conjurer le péril de ne pas contrôler son destin.

$\overline{3}$ Philippe d'Iribarne, Penser la diversité du monde, op. cit. 


\section{Une grande diversité de visions des rapports entre clients et fournisseurs}

Parmi les rapports entre acteurs économiques, les rapports entre clients et fournisseurs apparaissent comme ceux qui obéissent le plus évidemment à une logique contractuelle. Ils constituent à ce titre un bon terrain pour observer l'influence des cultures sur la conception de ces rapports. Nous en donnerons trois exemples. Le premier concerne la variété des manières dont les clients d'une banque de développement conçoivent leurs rapports avec celle-ci. Le deuxième porte sur la manière dont une entreprise internationale présente ses relations avec ses clients d'une part dans une perspectives française et d'autre part dans une perspective américaine. Le troisième porte sur les relations entre clients et fournisseurs dans les rapports entre entreprises en Chine.

Une banque de développement et ses clients

Les rapports entre une banque de développement et ses emprunteurs, publics et privés, relèvent d'une sphère financière qui fonctionne à une échelle largement planétaire. Et pourtant une recherche portant sur les attentes des emprunteurs a montré que, d'une société à l'autre, ceux-ci conçoivent très diversement leurs rapports avec une telle banque. Elle a de plus mis en évidence le fait que, au sein d'une même société, on trouve une grande convergence entre les visions des emprunteurs, même si ceux-ci appartiennent à des institutions très diverses, sont hauts-fonctionnaires, petits entrepreneurs ou banquiers locaux. Cette différence et ces convergences apparaissaient nettement dans les propos, dont nous allons donner quelques exemples, qui nous ont été tenus par nos interlocuteurs au cours de cette recherche ${ }^{4}$.

$4 \quad$ On s'appuiera sur les résultats d'une enquête faite pour l'Agence Française de Développement (AFD) dans 7 pays ou territoires auprès d'un ensemble de ses partenaires publics ou privés. Les entretiens évoqués dans ce texte ont été réalisés en Afrique du sud et au Vietnam par Jean-Pierre Segal, au Tchad par Sylvie Chevrier, en Nouvelle-Calédonie par Hèla Yousfi. Les résultats de la recherche sont présentés dans L'AFD et ses partenaires : la dimension culturelle (Philippe d'Iribarne, Paris, Agence française de développement (AFD), Document de travail, $\left.\mathrm{n}^{\circ} 23,2006\right)$. 
Dans l'univers anglo-saxon, rencontré en Afrique du Sud où l'establishment blanc reste dominant au sein des institutions financières, il paraît clair à tous que prêteurs et emprunteurs se rencontrent pour faire des affaires. Même lorsqu'il est question de développement et d'aide aux plus démunis, il s'agit toujours de "business ". La concurrence entre prêteurs d'un côté, entre emprunteurs de l'autre constitue le grand régulateur. La seule manière pour un prêteur de s'imposer est d'être plus attractif que ses concurrents, de trouver "une solution plus compétitive ». Il ne suffit pas d'avoir de bons produits, mais de savoir les vendre, faire son "marketing». Il n'est pas question de chercher à changer les autres, mais de savoir ce qu'on peut en attendre pour décider ce que l'on va faire soi-même. "Vous évaluez la capacité du management à faire ou non quelque chose [...]. Une fois que vous êtes au clair, vous décidez si vous prêtez ou non ". L'existence d'agences de certification aide chaque prêteur à évaluer les risques qu'il prend. Il est bon d'être "une organisation dotée d'une bonne réputation, bien notée par des agences internationales ». La référence à de bonnes relations humaines n'est pas absente. Mais c'est un ingrédient du bon fonctionnement d'un marché : "Si nous avons une idée pour les organismes qui financent le développement, nous la partageons avec chacun d'eux. [...]. S'ils y trouvent quelque intérêt, nous passons à l'étape suivante. Cela peut ne pas être explicite mais implicitement c'est de la coopération ".

On pourrait être tenté de dire qu'il s'agit de relations universelles, entre un banquier et ses clients au sein d'une économie de marché. Mais en fait, dès qu'on sort du monde anglo-saxon, on s'aperçoit que d'autres manières de raccorder les intérêts et les points de vue font référence.

Arrivant dans un univers français, abordé, dans le cadre de cette recherche, en Nouvelle-Calédonie où la plupart de nos interlocuteurs étaient des Français dits "de métropole », présents de manière transitoire, ce n'est plus la rencontre sur un marché qui est mise en avant. C'est la construction d'une relation où chacun agit conformément à ce qu'on peut attendre de quelqu'un qui occupe la place qui est la sienne dans la société, place évoquée 
par nos interlocuteurs en utilisant une vaste gamme d'images. Pour savoir ce qu'on peut attendre d'un partenaire, la question centrale est de savoir quel est son métier, sa mission, sa vocation. Dans le cas qui nous occupe on trouvait à un pôle l'image à la fois majestueuse, désintéressée et plutôt arrogante de l'État et de ses représentants, "une grande dame qui ne personnalise pas trop ses relations " et à l'opposé celle du commerçant qui démarche ses clients, "le Chinois du coin qui veut développer son commerce". D'autres images encore émaillaient les propos, telle celle du "prêteur sur gages" qui refuse de prendre des risques. Les exigences associées à la place que chacun occupe définissent ce qui est " normal ", " logique " d'en attendre. "Ils nous imposent des choses mais c'est logique, on aimerait bien faire le reproche mais on ne peut pas le faire, parce que c'est normal de demander des justifcatifs et tout ça... " La rencontre idéale se produit quand le fournisseur cherchant à exceller selon les normes de son métier, en l'exerçant de manière à la fois intelligente et inventive, s'intéresse aux attentes de son client et lui procure bien mieux qu'un produit standard. "On a fait part de nos besoins, les services du siège ont travaillé sur le sujet, et puis ont pu présenter un produit qui était tout à fait adapté à ce qu'on demandait. [...] C'est un produit financier qu'il fallait inventer. Mais ça correspond exactement à ce dont on avait besoin. [...] Aujourd'hui l'AFD c'est un partenaire de développement."

Chez nos interlocuteurs vietnamiens, il était fait constamment référence à ce qui est bien en soi, selon des critères qui transcendent les désirs de ceux qui sont immédiatement concernés. "Vous êtes sur la bonne voie "; "L'argent prêté est utilisé à un bon objectif»; "Un bénéficiaire doit utiliser les fonds de façon efficace et fructueuse ». Ce n'est pas que chacun suive spontanément cette bonne voie et le risque est grand que chaque membre ordinaire de la société, cherchant à tirer parti de la situation, détourne les choses du cours qu'elles devraient suivre. "Le bénéficiaire n'est pas conscient de sa responsabilité". "Je crois que les bénéficiaires du projet vont utiliser cela à un but différent". Il revient à ceux qui exercent un pouvoir de lutter contre ces dérives. "L'objectif des 
bénéficiaires est de tirer de l'argent du prêt, [...] mais nous [c'est un officiel qui parle] c'est de gérer le plus efficacement possible». Au nom de cette vision du bien, il est légitime de critiquer ceux avec qui on coopère si l'on considère qu'ils s'égarent. "Ce projet, on peut dire que l'AFD n'a pas compris la situation et cela a conduit à la non-réussite du projet" mais le risque d'affrontements violents est combattu en tempérant en permanence les critiques portant sur les actions par des propos aimables portant sur les personnes, propos reconnaissant leurs efforts, la qualité de leur implication, leur travail et leurs progrès. "L'AFD a fait beaucoup d'efforts et de progrès ces derniers temps". Et il est sans cesse affirmé que, si l'on critique autrui, on a aussi à s'améliorer soi-même. "On ne peut pas dire à qui est la faute; on pourrait améliorer".

Dans la vision des choses évoquée par nos interlocuteurs tchadiens, chaque emprunteur a en charge la défense de ses intérêts. On ne peut trop compter sur des représentants ou des relais, suspects d'être tentés d'agir pour leur propre compte. "Les gens pensent que sils gèrent un projet, ils vont avoir des indemnités, ils vont en profiter. "Mais les processus sur lesquels on compte pour permettre aux prêteurs et aux emprunteurs de s'ajuster sont bien différents de ceux qui font référence dans le monde anglosaxon. Il n'est pas question de simples relations d'affaires, avec ce qu'ils supposent d'impersonnalité. Il est nécessaire d'avoir des rapports directs avec ceux avec qui on est amené à coopérer, rapports sans lesquels on ne peut saisir précisément leurs attentes. "Nous avons mis en place une relation plus poussée, des relations de collaboration très étroite avec l'AFD en ce qui concerne des correspondances, des réunions, des rapports physiques avec l'AFD. " L'ajustement entre les intérêts des parties est vu comme le fruit d'une confrontation patiente des points de vue, grâce à laquelle chacun devient conscient de ce à quoi tiennent ses partenaires et on arrive finalement à un compromis. S'il n'y a "pas assez de place à la concertation ", les décisions prises risquent de se révéler " complètement inadaptées". Les relations qui s'instaurent entre partenaires habituels conduisent à se déclarer " amis " : "je suis arrivé à avoir à titre personnel des rapports qui sont très bons, qui 
sont très cordiaux avec tous ces gens là y compris les collègues, les amis de Proparco ${ }^{5}$, les amis de la BEI $"$. Mais ce n'est pas parce que l'on est " amis " que, dans le processus d'ajustement entre les intérêts, on ne va pas chercher à tirer le maximum de la situation. "J'ai obtenu beaucoup de choses de Proparco parce que de l'autre, j'ai la DEG ${ }^{7}$ et la BEI. [...] Si l'une cède et l'autre résiste, avec la menace quion peut se passer d'elle, elle s'aligne. On a obtenu des choses comme ça."

Quand on rapproche les quatre visions que nous venons d'évoquer, on voit combien une "même " situation (en l'occurrence les rapports entre une banque de développement et ses clients) peut être gérée de manières diverses selon les lieux. Dans certains cas, comme dans le monde anglo-saxon ${ }^{8}$ ou au Tchad, chacun peut défendre ses intérêts en toute légitimité sans avoir à se référer à une vision du bien réputée transcender les points de vue des parties. C'est le respect d'une forme de procédure permettant un ajustement mutuel qui fait référence. On a ainsi une approche procédurale du bien commun. Dans d'autres cas, comme en France ${ }^{9}$ ou au Vietnam, une certaine notion de ce qui est bien en soi fait référence et vient interférer avec la façon dont chacun défend ses intérêts. On a ainsi une vision substantielle du bien commun. Simultanément, il existe de multiples options à l'intérieur des familles ainsi définies : le marché ou une sorte de palabre comme formes de procédures; l'excellence dont chacun est en quête dans l'accomplissement de son métier ou la bonne voie dont les détenteurs du pouvoir sont les gardiens comme expression d'une vision substantielle du bien.

Filiale de l'Agence française de développement tournée vers les entreprises.

6 Banque européenne d'investissement.

7 Deutsche Investitions- und Entwicklungsgesellschaft (Banque allemande de développement)

8 Dans le cadre de cette recherche, nous avons rencontré ce monde dans l'establishment bancaire blanc d'Afrique du Sud, mais ce que nous y avons observé rejoint un ensemble d'observations faites de manière plus générale dans ce monde (Philippe d'Iribarne, La logique de l'honneur, op. cit. et Penser la diversité du monde, op. cit.).

9 Pour laquelle on peut faire la même remarque que celle qui précède concernant le monde anglo-saxon. 


\section{Client français, client américain}

Deux versions de référence des Principes d'action d'un groupe industriel, Lafarge, l'une française et l'autre américaine, se correspondent strictement, phrase à phrase. Elles proclament les mêmes valeurs, et utilisent souvent les mêmes mots (satisfaction, satisfaction; environnement, environment; etc.). Pourtant, un examen plus attentif laisse apparaître un grand nombre de différences qui ne doivent rien au hasard ${ }^{10}$. C'est que le texte ne se contente pas de faire référence à des valeurs. Il rentre dans le concret, évoque les rapports que l'entreprise entend nouer avec ses clients, ses actionnaires et son personnel. En prêtant attention à la façon dont chacune des deux versions évoque l'action de l'entreprise, aux images dont elle se sert à cet effet, on voit que chacune d'elles est marquée par une vision particulière d'une bonne manière de vivre et d'œuvrer ensemble. Il en est ainsi en particulier pour les rapports entre l'entreprise et ses clients.

Observons deux passages concernant ces rapports. Provide the construction industry / " offrir au secteur de la construction "; delivering the [...] products... / " proposer les produits..." . Linguistiquement, rien n'aurait empêché d'écrire en français " fournir " plutôt qu' " offrir " dans le premier cas et "livrer " plutôt que "proposer" dans le second. Mais la manière de mettre en scène les rapports entre l'entreprise et ses clients aurait été profondément modifiée. Provide et delivering évoquent un rapport marchand entre un donneur d'ordre (le client) qui a passé commande et un prestataire (l'entreprise) qui fournit le produit commandé. Cette représentation suggère une forme de relation qui paraît fondamentalement positive dans une perspective américaine : un rapport contractuel entre individus indépendants dont les intérêts se rencontrent. Au contraire, les équivalents français, "fournir », " livrer ", des termes employés dans la version américaine, évoquent pour des Français une activité mercantile, à l'honorabilité douteuse. "Offrir » et " proposer " suggèrent une

$10 \quad$ Philippe d'Iribarne, L'envers du moderne. Conversation avec Julien Charnay, Paris, CNRS éditions, 2012 et Philippe d'Iribarne, L'épreuve des différences, op. cit. 
autre forme de rapports, plus désintéressés et donc plus honorables.

Certes, il n'est pas question que l'entreprise offre ses produits, ou les propose, au sens où elle en ferait cadeau. Il est sous entendu qu'elle les offre pour de l'argent. Mais le terme offrir, comme celui de proposer ne sont pas pour autant des fauxsemblants. Ce qu'il s'agit d'offrir n'est pas une marchandise ordinaire : cela est constitué, affirme le texte des Principes, par "les produits, systèmes et solutions les plus fiables, les plus innovants et les plus économiques ". L'entreprise ne se contente pas de livrer ces produits, comme elle pourrait le faire pour un produit standard : elle les conçoit, fait qu'ils existent, les rend disponibles pour leur utilisateurs potentiels. Et il est bien vrai que cette existence est en quelque sorte " offerte ». Les termes " offrir ", " proposer " sont associés au fait que l'attention est dirigée moins vers la phase, peu noble, où les produits sont finalement vendus que vers la phase, beaucoup plus noble, où ils sont conçus. Il y a bien dans cette phase une sorte d'amour de l'art, une certaine gratuité, qui écarte toute assimilation à une activité servile.

Simultanément là où, dans la version américaine, l'entreprise apparaît comme soumise aux attentes de ses clients, elle est présentée, dans la version française, comme décidant souverainement des rapports qu'elle entretient avec eux. Comparons à cet égard : Being a customer driven organization / "Orienter notre organisation vers le client "; Being measured by our customer's satisfaction and loyalty / "Faire du niveau de satisfaction de nos clients et de leur fidélité la mesure de notre succès ». Dans la version américaine, c'est le client qui est moteur. L'entreprise est conduite (driven) par la volonté de celui-ci. Sa valeur dépend de la façon dont le client la considère et la traite; l'entreprise ne peut que constater que les réactions du client fournissent l'aune qui sert à la "mesurer ». Cette manière de voir les choses apparaît comme parfaitement satisfaisante dans une société où il va de soi que l'on travaille pour quelqu'un d'autre, où il s'agit de répondre à ses attentes et où c'est au marché, à l'issue d'une compétition 
loyale, de déterminer ce que l'on vaut. Dans une vision française, une telle manière d'être à la remorque de ses clients, voire à leur botte, apparaîtrait comme peu digne. Par contre, décider librement de "s'orienter " vers le client, dans une sorte de démarche souveraine dont on garde l'initiative, est compatible avec une relation digne où, le considérant avec bienveillance, on veille sur lui. De même, il est parfaitement digne de décider souverainement que, s'intéressant à ce qu'éprouvent ses clients, on va en " faire " un repère pour orienter son action.

On peut rapprocher cette approche française du fait que, de manière générale, on a en France toute une manière de parler du rapport au client en utilisant des termes - prescrire, diagnostiquer, accueillir, etc. - qui mettent en scène ce rapport en le reliant à une expérience de grandeur désintéressée et non de servilité mercantile.

\section{Clients et fournisseurs en Chine}

L'influence de la culture sur la conception des relations contractuelles entre clients et fournisseurs en Chine a été analysée de manière spécifique à l'occasion d'une thèse, que j'ai dirigée, portant sur la mise en ouvre de contrats commerciaux dans les rapports entre entreprises ${ }^{11}$.

Dans ce cas, la coexistence entre d'un côté le fait que les clauses des contrats sont souvent peu respectées et que l'on ne peut guère se confier à la justice pour obtenir réparation, et de l'autre le fait que les entreprises font un large usage de contrats extrêmement précis, fait question. L'explication est liée au fait qu'un contrat entre entreprises n'est en général qu'un épisode d'une relation à long terme, ou les créances et les dettes ne s'équilibrent pas à chaque instant mais seulement dans la durée. Des propos tenus par un vendeur traduisent bien ce qu'il en est :

Une fois un de mes clients avec lesquels nous avons une longue histoire de coopération s'est plaint de la mauvaise qualité de nos produits et m'a

11 Mingming Duan, Incomplétude des contrats et relations inter-firmes dans une économie en transition : le cas de la Chine, thèse, Université Paris X Nanterre, 2007. 
demandé d'aller sur place pour régler le problème. Je me suis déplacé au rendez-vous pour parler en toute sincérité du problème. Il faut d'abord clarifier la responsabilité. Si c'est la mienne, je vais récupérer toutes les marchandises et les changer. Si c'est la sienne, j'envisage également des compromis. Finalement c'était la sienne, c'est-à-dire qu'il manquait d'entretien nécessaire. J'ai quand même décidé de leur changer les pièces en question. Pourquoi ? Parce que je regarde notre relation à long terme. Si j'avais révélé que c'était à cause des défaillances du service d'entretien que les pièces détachées se sont mises rapidement hors service, les vrais responsables auraient subi des reproches de leur hiérarchie. Dans ce cas, ils auraient manifesté des mécontentements vis-à-vis de moi, alors que c'est avec eux et non (directement) avec leurs hiérarchies que je travaille. $\mathrm{Si}$ je les mets dans une situation très embarrassante, ce sera difficile pour moi de les voir la prochaine fois : je risque de les perdre comme clients. Au contraire, si j'assume volontiers la responsabilité qui n'est pas la mienne, ces vrais responsables vont avoir un sentiment de reconnaissance très fort vis-à-vis de moi parce qu' ils me doivent quelque chose. Ce contexte est très favorable pour le renforcement de nos relations, la communication sera par la suite plus facile, on se comprendra mieux. Cet attachement sentimental se traduira sans doute par plus de commandes après, plus de fidélité, plus de loyauté. Nos destins seront liés plus étroitement ${ }^{12}$.

L'écart entre ce qui est prévu dans un contrat et ce qui est advenu en fin de compte, tant en matière de délais, que de qualité ou de paiement, prend sens dans une telle perspective. Le fait de s'être entendu sur un contrat précis permet non d'obtenir un "enforcement" de ses clauses, ou des pénalités en cas de non respect de celles-ci, mais de savoir qui est en dette morale à l'égard de qui et quelle est l'ampleur de la dette.

\section{Conception contractuelle des rapports économiques et culture américaine}

La conception contractuelle des rapports entre agents économiques sous-tend tellement les théories du management et les théories économiques qu'elle pourrait paraître aller de soi; être quelque chose d'inhérent, de manière universelle, à un fonctionnement efficace d'une entreprise et plus largement des rapports

$\overline{12}$ Mingming Duan, "Contrats et culture dans les relations interentreprises en

Chine ", Management international, vol. 14, n 2, 2010, p. 124-125. 
économiques. En fait, quand on examine les raisons pour lesquelles cette conception joue un tel rôle aux États-Unis, lieu où sont conçues la plupart des théories du management et où prospère le mainstream de la pensée économique, il apparaît bien que les préoccupations d'efficacité sont loin d'être seules en cause.

L'attachement américain à une conception contractuelle se manifeste par le fait qu'elle conduit à résister à la mise en place de formes d'organisation dont les Américains eux-mêmes reconnaissent qu'elles sont plus efficaces que des rapports strictement contractuels. Ceci est bien apparu, par exemple, au début des années 1990, quand les succès japonais dans l'industrie automobile ont conduit à prôner la mise en place aux États-Unis d'un mode d'organisation de la production (lean production) d'inspiration japonaise. Une vaste étude sur l'industrie automobile dans le monde menée par le Massachusetts Institute of Technology (MIT) a pris comme postulat que ce mode d'organisation pouvait et devait être mis en œuvre sur toute la planète, indépendamment des cultures ${ }^{13}$. Or les auteurs ont été obligés de constater l'existence, aux États-Unis, d'un écart entre l'enthousiasme de principe pour certaines pratiques qu'ils préconisaient et les réticences de fait par rapport à ces pratiques. Cet écart était spécialement net pour la manière d'organiser les relations entre les entreprises et leurs sous-traitants. L'organisation traditionnelle de ces relations aux États-Unis (le client définit un cahier des charges sans que les candidats fournisseurs s'en mêlent, les candidats fournisseurs font une offre sans que le client se mêle de leur fonctionnement interne, puis le client choisit et un contrat est passé) met en œuvre une manière de concevoir les relations entre les partenaires qui laisse chacun d'eux maître de ses décisions. Leur coopération est fondée sur l'accord, avec des clauses bien définies précisant les obligations et les droits des parties, de leurs volontés souveraines. Au contraire, la forme d'organisation d'inspiration japonaise reconnue comme plus efficace interdit de définir sans

13 James P. Womack, Daniel T. Jones, Daniel Roos, The Machine that Changed the World, New-York et Toronto, Rawson Associates, Collier Macmillan Canada et Maxwell Macmillan international, 1990. 
ambiguïté qui est responsable de quoi; ainsi les sous-traitants sont tenus au courant très en amont des projets de leur client, euxmêmes laissent leur client examiner de près leur processus de production et connaître leurs prix de revient ${ }^{14}$. C'est ce fait que chacun n'est plus maître de son destin qui est mal accepté dans un contexte américain.

De même quand on passe des théories du management à l'observation du fonctionnement interne d'une entreprise américaine, il apparaît que la mise en œuvre de rapports contractuels pour gérer les relations entre une entreprise et chaque membre de son personnel rencontre mainte difficulté; il n'est pas facile d'y avoir recours pour formater une vie des organisations qui s'inscrit souvent mal dans ce cadre. Il faut en effet, pour mettre ces rapports en ouvre, représenter par quelques chiffres les fruits de la contribution de chacun à l'œuvre commune. Or ceci n'est que rarement possible en toute rigueur et parfois est gravement caricatural. Cette constatation a été au cour des interrogations relatives à l'efficacité des méthodes de management couramment pratiquées qui ont marqué les États-Unis au cours des années $1980^{15}$. L'attachement à cette approche apparaît moins fondé sur des considérations d'efficacité que sur le mode de relation qu'elle instaure entre l'entreprise et ceux qui y travaillent et, au premier chef, sur la forme d'autonomie qu'elle accorde à ceux-ci. Chacun, maître de déterminer la manière de s'y prendre pour répondre à la commande qu'il doit satisfaire, a le sentiment que personne d'autre que lui ne va régir son existence :

Je pense que si les résultats [the bottom line numbers], les résultats sont là, affirme par exemple un manager, la manière dont vous les avez obtenus est dans ces conditions ok. La plupart des gens pensent qu'ils doivent être quelque peu maîtres de leur propre destin, et déterminent comment ils viennent à bout de leur travail quelque peu tous seuls. Aussi

\footnotetext{
14 Masahiko Aoki, "Toward an Economic Model of the Japanese Firm », Journal of Economic Literature, vol. 28, n ${ }^{\circ}$ 1, p. 1-27.

15 William G. Ouchi, Theory Z: How American Business Can Meet the Japanese Challenge, Reading (Mass.) et Don Mills (Ont.), Addison-Wesley, 1981; Peters, Thomas J. et Robert H. Waterman Jr, In Search of Excellence: Lessons from America's Best-Run Companies, New York, Harper \& Row, 1982.
} 
longtemps que les objectifs sont atteints, les supérieurs doivent être satisfaits ${ }^{16}$.

Une telle conception contractuelle des rapports de travail, qui marque l'histoire des États-Unis est en rapport avec une vision particulière des rapports de subordination. Ainsi, note Tocqueville à propos des rapports entre maître et serviteur, "Pourquoi donc le premier [le maître] a-t-il le droit de commander et qu'est-ce qui force le second [le serviteur] à obéir ? L'accord momentané et libre de leurs deux volontés. [...] Le maître juge que dans le contrat est la seule cause de son pouvoir, et le serviteur y découvre la seule clause de son obéissance ${ }^{17}$ ".

Cet attachement à des rapports contractuels s'inscrit dans une culture politique qui y voit le meilleur rempart contre l'arbitraire et par là contre la servitude. Ainsi, Locke oppose l'homme libre qui, tout en travaillant pour un autre, est lié à lui par un rapport contractuel définissant précisément ses droits et obligations à l'esclave soumis à un pouvoir arbitraire :

Un homme libre se rend serviteur et valet d'un autre, en lui vendant, pour un certain temps, son service, moyennant un certain salaire. Or, quoique cela le mette communément dans la famille de son maître, et l'oblige à se soumettre à sa discipline et aux occupations de sa maison, il ne donne pourtant de pouvoir au maître sur son serviteur ou son valet, que pendant quelque temps, que pendant le temps qui est contenu et marqué dans le contrat ou le traité fait entre eux. Mais il y a une autre sorte de serviteurs, que nous appelons, d'un nom particulier, esclaves, et qui $[. .$.$] sont [\ldots]$ sujets à la domination absolue et au pouvoir arbitraire de leurs maitres ${ }^{18}$.

Dans une société régie par des rapports contractuels, autrui ne peut rien m'imposer, puisqu'il ne peut rien exiger de moi si ce n'est ce à quoi j'ai consenti en ratifiant le contrat qui nous lie.

16 Philippe d'Iribarne, La logique de l'honneur, op. cit., p. 138.

17 Alexis de Tocqueville De la démocratie en Amérique, tome II, 1840, troisième partie, chapitre V.

18 John Locke, Two Treatises of Government, édité par Peter Laslett, Cambridge University Press, 1960 [1690]; traduction française, limitée au deuxième traité, Traité du gouvernement civil, Garnier-Flammarion, 1992, $\$ 85$. 


\section{Conclusion}

Dès lors que l'on prête attention à ce que les acteurs entendent faire lorsqu'ils s'engagent dans une relation contractuelle, au sens qu'ils donnent aux liens entre personnes qui se nouent à ce propos, on voit combien ils tendent à construire cette relation d'une manière telle qu'elle s'accorde à ce qui apparaît, dans leur culture, comme une manière satisfaisante de vivre et travailler ensemble. On ne peut isoler un univers contractuel qui aurait ses lois propres, indépendantes de l'organisation de la vie de la société.

Quand la représentation de la société qui prévaut au sein des approches économiques y voit pour l'essentiel un ensemble de rapports contractuels, la faible compréhension de cet encastrement culturel du fonctionnement des contrats alimente la faible compréhension de l'encastrement culturel de l'économie globale. Cette situation est entretenue par une sorte de cercle vicieux. Le fait qu'on dispose de peu de données empiriques mettant précisément en évidence les mécanismes qui sont à l'œuvre dans cet encastrement culturel favorise le maintien d'une vision universaliste de l'économie, vision qui elle-même entretient la clôture disciplinaire de celle-ci et l'absence de travaux situés à l'interface de l'économie et des autres sciences de l'homme et de la société. Les multiples déclarations d'économistes éminents, en particulier lors de conférences Nobel ou d'adresses présidentielles aux grands congrès d'économistes, invitant la discipline à une meilleure prise en compte du monde réel, ne modifient guère cette situation. Et cet enfermement disciplinaire ne fait pas qu'entraver le progrès des connaissances. Il constitue un obstacle à ce que soient mises en place des institutions encadrant le fonctionnement de l'économie qui seraient rendues efficaces par une prise en compte de la réalité des sociétés considérées dans leur diversité. Cela concerne de multiples institutions, depuis celles qui régulent le fonctionnement de la concurrence jusqu'à celles qui permettent de lutter contre la corruption. Un vaste champ d'investigations, de grande importance à la fois théorique et pratique, s'ouvre ainsi devant nous. 


\section{Bibliographie}

Aoki, Masahiko, "Toward an Economic Model of the Japanese Firm ", Journal of Economic Literature, vol. 28, n ${ }^{\circ}$ 1, p. 1-27.

Duan, Mingming, Incomplétude des contrats et relations inter-firmes dans une économie en transition : le cas de la Chine, thèse, Université Paris X Nanterre, 2007.

Duan, Mingming, "Contrats et culture dans les relations interentreprises en Chine ", Management international, vol. 14, $\mathrm{n}^{\circ}$ 2, 2010, p. 121-129.

Iribarne, Philippe (d'), L'AFD et ses partenaires : la dimension culturelle, Paris, Agence française de développement (AFD), Document de travail, $\mathrm{n}^{\circ} 23,2006$.

Iribarne, Philippe (d'), L'envers du moderne. Conversation avec Julien Charnay, Paris, CNRS éditions, 2012.

Iribarne, Philippe (d'), L'épreuve des différences. L'expérience d'une entreprise mondiale, Paris, Seuil, coll. "La couleur des idées », 2009.

Iribarne, Philippe (d'), La logique de l'honneur. Gestion des entreprises et traditions nationales, Paris, Seuil, 1989.

Iribarne, Philippe (d'), Penser la diversité du monde, Paris, Seuil, 2008.

Iribarne, Philippe (d'), Alain Henry, Jean-Pierre Segal, Sylvie Chevrier, Tatjana Globokar, Cultures et mondialisation. Gérer par-delà les frontières, Paris, Seuil, 1998.

Locke, John, Two Treatises of Government, édité par Peter Laslett, Cambridge University Press, 1960 [1690]; traduction française, limitée au deuxième traité, Traité du gouvernement civil, Garnier-Flammarion, 1992.

Ménard, Claude (dir.), Institutions, Contracts and Organizations. Perspective from New-Institutional Economics, Cheltenham (UK) et Northampton (MA), Edward Elgar, 2000.

Ouchi, William G., Theory Z: How American Business Can Meet the Japanese Challenge, Reading (Mass.) et Don Mills (Ont.), Addison-Wesley, 1981.

Peters, Thomas J. et Robert H. Waterman Jr, In Search of Excellence : Lessons from America's Best-Run Companies, New York, Harper \& Row, 1982.

Tocqueville, Alexis (de), De la démocratie en Amérique, tome II, 1840.

Womack, James P., Daniel T. Jones, Daniel Roos, The Machine that Changed the World, New-York et Toronto, Rawson Associates, Collier Macmillan Canada et Maxwell Macmillan international, 1990. 